\title{
Multi-adaptive Natural Language Generation using Principal Component Regression
}

\author{
Dimitra Gkatzia, Helen Hastie, and Oliver Lemon \\ School of Mathematical and Computer Sciences, Heriot-Watt University, Edinburgh \\ $\{$ dg106, h.hastie, o.lemon\}@hw.ac.uk
}

\begin{abstract}
We present FeedbackGen, a system that uses a multi-adaptive approach to Natural Language Generation. With the term 'multi-adaptive', we refer to a system that is able to adapt its content to different user groups simultaneously, in our case adapting to both lecturers and students. We present a novel approach to student feedback generation, which simultaneously takes into account the preferences of lecturers and students when determining the content to be conveyed in a feedback summary. In this framework, we utilise knowledge derived from ratings on feedback summaries by extracting the most relevant features using Principal Component Regression (PCR) analysis. We then model a reward function that is used for training a Reinforcement Learning agent. Our results with students suggest that, from the students' perspective, such an approach can generate more preferable summaries than a purely lecturer-adapted approach.
\end{abstract}

\section{Introduction}

Summarisation of time-series data refers to the task of automatically generating reports from attributes whose values change over time. Content selection is the task of choosing what to say, i.e. what information is to be included in a report (Reiter and Dale, 2000). We consider the task of automatically generating feedback summaries for students describing their performance during the lab of a computer science module over the semester.

Various factors can influence students' learning such as difficulty of the material (Person et al., 1995), workload (Craig et al., 2004), attendance in lectures (Ames, 1992), etc. These factors change over time and can be interdependent.
In addition, different stakeholders often have conflicting goals, needs and preferences, for example managers with employees, or doctors with patients and relatives, or novice and expert users. In our data, for instance, lecturers tend to comment on the hours that the student studied, whereas the students disprefer this content. In our previous work, we showed that lecturers and students have different perceptions regarding what constitutes good feedback (Gkatzia et al., 2013). Here, we present a novel approach to generation by adapting its content to two user groups simultaneously. Producing the same summary for two groups is important as it allows for shared context and meaningful further discussion and reduces development time.

\section{Related Work}

Previous work on NLG systems that address more than one user group employs different versions of a system for each different user group (Gatt et al., 2009; Hunter et al., 2011; Mahamood and Reiter, 2011), makes use of User Models (Janarthanam and Lemon, 2010; Thompson et al., 2004; Zukerman and Litman, 2001) or personalises the output to individual users using rules (Reiter et al., 1999). Our proposed system adapts the output to the preferences of more than one user type ${ }^{1}$, lecturers and students, but instead of developing many different systems or using User Models that describe different users, it attempts to model the middle ground between the preferences.

In order to identify the users' preferences, we apply Principal Components Regression (PCR (Jolliffe, 1982)) analysis to two datasets that contain lecturers' and students' ratings and identify the most important variables from the principal components, which are then included in a reward function. This hand-crafted reward function is used for training an RL agent for summarisation \footnotetext{
tion.
} 
Raw Data

\begin{tabular}{lllll}
\hline factors & week 2 & week 3 & $\ldots$ & week 10 \\
\hline marks & 5 & 4 & $\ldots$ & 5 \\
hours_studied & 1 & 2 & $\ldots$ & 3 \\
$\ldots$ & $\ldots$ & $\ldots$ & $\ldots$ & $\ldots$ \\
\hline
\end{tabular}

Trends from Data

\begin{tabular}{ll}
\hline factors & trend \\
\hline (1) marks & trend_other \\
(2) hours_studied & trend_increasing \\
(3) understandability & trend_decreasing \\
(4) difficulty & trend_decreasing \\
(5) deadlines & trend_increasing \\
(6) health_issues & trend_other \\
(7) personal_issues & trend_decreasing \\
(8) lectures_attended & trend_other \\
(9) revision & trend_decreasing \\
\hline
\end{tabular}

Summary

Your overall performance was excellent during the semester. Keep up the good work and maybe try some more challenging exercises. Your attendance was varying over the semester. Have a think about how to use time in lectures to improve your understanding of the material. You spent 2 hours studying the lecture material on average. You should dedicate more time to study. You seem to find the material easier to understand compared to the beginning of the semester. Keep up the good work! You revised part of the learning material. Have a think whether revising has improved your performance.

Table 1: The table on the top left shows an example of the time-series data. The table on the bottom left shows an example of described trends. The box on the right presents a target summary.

of time-series data. Our previous work showed that when comparing RL and supervised learning in the context of student feedback generation, students preferred the output generated by the RL system (Gkatzia et al., 2014a). Therefore, here, we used RL rather than a supervised learning method. The work described here builds on work reported in (Gkatzia et al., 2014b), which uses as a reward function the average of the Lecturer-adapted and Student-adapted reward functions. However, that method seems to cancel out the preferences of the two groups whereas PCR is able to identify relevant content for both groups.

In the next section, we describe the data used, and the methodology for the multi-adaptive NLG, as well as two alternative systems. In Section 4, we describe the comparison of these three systems in a subjective evaluation and present the results in Section 5. A discussion follows in Section 6 and finally, future work is discussed in Section 7.

\section{Methodology}

Reinforcement Learning is a machine learning technique that defines how an agent learns to take optimal sequences of actions so as to maximize a cumulative reward (Sutton and Barto, 1998). In our framework, the task of summarisation of timeseries data is modelled as a Markov Decision Process, where the decisions on content selection cor- respond to a sequence of actions (see Section 3.2). Temporal Difference (TD) learning (Sutton and Barto, 1990) is used for training three agents in a simulated environment to learn to make optimal content selection decisions:

1. by adapting to both groups simultaneously,

2 . by adapting to lecturers,

3. by adapting to students.

\subsection{The Data}

For this study, the dataset described in (Gkatzia et al., 2013) was used. Table 1 presents an example of this dataset that describes a student's learning factors and an aligned feedback summary provided by a lecturer. The dataset is composed of 37 similar instances. Each instance consists of time-series information about the student's learning routine and the selected templates that lecturers used to provide feedback to this particular student. A template is a quadruple consisting of an id, a factor (bottom left of Table 1), a reference type (trend, week, average, other) and surface text. For instance, a template can be (1, marks, trend, "Your marks were $<$ trend $>$ over the semester"). The lexical choice for $<$ trend $>$ (i.e. increasing or decreasing) depends on the values of time-series data. There is a direct mapping between the values of factor 
and reference type and the surface text. The timeseries factors are listed in Table 1.

\subsection{Actions and states}

The state consists of the time-series data and the number of factors which have so far been selected to be talked about (the change of the value of this variable consequently introduces a state change). In order to explore the state space the agent selects a time-series factor (e.g. marks, deadlines etc.) and then decides whether to talk about it or not, until all factors have been considered.

\subsection{Reward function}

The reward function is the following cumulative multivariate function:

$$
\text { Reward }=a+\sum_{i=1}^{n} b_{i} * x_{i}+c * \text { length }
$$

where $X=\left\{x_{1}, x_{2}, \ldots, x_{n}\right\}$ describes the chosen combinations of the factor trends observed in the time-series data and a particular template (i.e. the way of mentioning a factor). $a, b$ and $c$ are the correlation coefficients and length describes the number of factors selected to be conveyed in the feedback summary. The value of $x_{i}$ is given by the function:

$$
x_{i}= \begin{cases}1, & \text { the combination of a factor trend } \\ 0, & \text { and a template type is included }\end{cases}
$$

The coefficients represent the level of preference for a factor to be selected and the way it is conveyed in the summary. In the training phase, the agent selects a factor and then decides whether to talk about it or not. If the agent decides to refer to a factor, the selection of the template is then performed in a deterministic way, i.e. it selects the template that results in higher reward.

Each rated summary is transformed into a vector of 91 binary features. Each feature describes both (1) the trend of a factor (e.g. marks increasing, see also Table 1) and (2) the way that this factor could be conveyed in the summary (e.g. one possible way is referring to average, another possible way is referring to increasing/decreasing trend). If both conditions are met, the value of the feature is 1 , otherwise 0 . The 91 binary features describe all the different possible combinations. For both the Lecturer-adapted and Studentadapted systems, the reward function is derived from a linear regression analysis of the provided dataset, similarly to Walker et al. (1997) and Rieser et al. (2010).

\subsubsection{Multi-adaptive Reward Function}

In order to derive a reward function that finds a balance between the two above mentioned systems, we use PCR to reduce the dimensionality of the data and thus reduce the introduced noise. Through PCR we are able to reduce the number of features and identify components of factors that are deemed important to both parties to be used in the reward function.

PCR is a method that combines Principal Component Analysis (PCA) (Jolliffe, 1986) with linear regression. PCA is a technique for reducing the dataset dimensionality while keeping as much of the variance as possible. In PCR, PCA is initially performed to identify the principal components, in our case, the factors that contribute the most to the variance. Then, regression is applied to these principal components to obtain a vector of estimated coefficients. Finally, this vector is transformed back into the general linear regression equation. After performing this analysis on both datasets (students and lecturers), we choose the most important (i.e. the ones that contribute the most to the variance) commoncomponents or features resulting in 18 features which were used in the reward function. We then design a handcrafted reward function taking into account this PCR analysis. The five most important features are shown in Table 2.

\begin{tabular}{|l|l|}
\hline factor trend & way it is mentioned \\
\hline (1) marks stable & average \\
(2) hours_studied decreasing & trend \\
(3) health_issues decreasing & weeks \\
(4) lectures_attended stable & average \\
(5) personal_issues increasing & trend \\
\hline
\end{tabular}

Table 2: The top 5 features out of the 18 selected through PCR analysis.

\section{Evaluation}

FeedbackGen is evaluated with real users against two alternative systems: one that adapts to lecturers' preferences and one that adapts to students' preferences. The output of the three systems is ranked by 30 computer science students from a variety of years of study. Time-series data of three students are presented on graphs to each participant, along with three feedback summaries (each one generated by a different system), in random order, and they are asked to rank them in terms of preference. 


\begin{tabular}{|c|c|c|}
\hline Student-adapted $\left\{\right.$ Ranking: $\left.1 \mathrm{st}^{*}\right\}$ & FeedbackGen $\{$ Ranking: 2nd*\} & Lecturer-adapted $\left\{\right.$ Ranking: $\left.3 \mathrm{rd}^{*}\right\}$ \\
\hline $\begin{array}{l}\text { You did well at weeks } 2,3,6,8,9 \text { and } 10 \text {, } \\
\text { but not at weeks } 4,5 \text { and } 7 \text {. Have a think } \\
\text { about how you were working well and } \\
\text { try to apply it to the other labs. Your at- } \\
\text { tendance was varying over the semester. } \\
\text { Have a think about how to use time in lec- } \\
\text { tures to improve your understanding of } \\
\text { the material. You found the lab exercises } \\
\text { not very challenging. You could try out } \\
\text { some more advanced material and exer- } \\
\text { cises. You dedicated more time study- } \\
\text { ing the lecture material in the beginning } \\
\text { of the semester compared to the end of } \\
\text { the semester. Have a think about what } \\
\text { is preventing you from studying. Revis- } \\
\text { ing material during the semester will im- } \\
\text { prove your performance in the lab. }\end{array}$ & $\begin{array}{l}\text { Your overall performance was } \\
\text { very good during the semester. } \\
\text { Keep up the good work and maybe } \\
\text { try some more challenging exer- } \\
\text { cises. You found the lab exer- } \\
\text { cises not very challenging. You } \\
\text { could try out some more advanced } \\
\text { material and exercises. You dedi- } \\
\text { cated more time studying the lec- } \\
\text { ture material in the beginning of } \\
\text { the semester compared to the end } \\
\text { of the semester. Have a think about } \\
\text { what is preventing you from study- } \\
\text { ing. You have had other dead- } \\
\text { lines during weeks } 6 \text { and } 8 \text {. You } \\
\text { may want to plan your studying and } \\
\text { work ahead. }\end{array}$ & $\begin{array}{l}\text { Your overall performance was very } \\
\text { good during the semester. Keep up the } \\
\text { good work and maybe try some more } \\
\text { challenging exercises. You found the } \\
\text { lab exercises not very challenging. You } \\
\text { could try out some more advanced mate- } \\
\text { rial and exercises. You dedicated more } \\
\text { time studying the lecture material in the } \\
\text { beginning of the semester compared to } \\
\text { the end of the semester. Have a think } \\
\text { about what is preventing you from study- } \\
\text { ing. You have had other deadlines during } \\
\text { weeks } 6 \text { and } 8 \text {. You may want to plan } \\
\text { your studying and work ahead. You did } \\
\text { not face any health problems during the } \\
\text { semester. You did not face any personal } \\
\text { issues during the semester. }\end{array}$ \\
\hline
\end{tabular}

Table 3: The table presents example outputs from the three different systems in order of highest ranked (bold signifies the chosen template content, $*$ denotes significance with $\mathrm{p}<0.05$ after comparing each system with each other using Mann Whitney U test).

\section{Results}

Table 3 shows three summaries that have been generated by the different systems. As we can see from Table 3, students significantly prefer the output of the system that is trained for their preferences. In contrast, students significantly disprefer the system that is trained for lecturers' preferences. Finally, they rank as second the system that captures the preferences of both lecturers and students, which shows that it might be feasible to find middle ground between the preferences of two user groups. Significance testing is done using a Mann Whitney $U$ test $(\mathrm{p}<0.05)$, performing a pair-wise comparison.

\section{Discussion}

The weights derived from the linear regression analysis vary from the Lecturer-adapted function to the Student-adapted function. For instance, the lecturers' most preferred content is hours_studied. This, however, does not factor heavily into the student's reward function, apart from the case where hours_studied are decreasing or remain stable (see also Table 2).

Students like reading about personal_issues when the number of issues they faced was increasing over the semester. On the other hand, lecturers find it useful to give advice to all students who faced personal issues during the semester, hencepersonal_issues are included in the top 18 features (Table 2). Moreover, students seem to mostly prefer a feedback summary that mentions the understandability of the material when it increases, which is positive feedback.

As reflected in Table 2, the analysis of PCR showed that both groups found it useful to refer to the average of marks when they remain stable. In addition, both groups found understandability when it increases useful, for a variety of reasons, for example lecturers might find it useful to encourage students whereas students might prefer to receive positive feedback. Both groups also agree on hours_studied as described earlier. On the other hand, both groups find mentioning the students' difficulty when it decreases as positive.

\section{Future Work}

In the future, we plan to evaluate our methodology with lecturers and a larger sample of students across different disciplines. Moreover, we aim to port our methodology to a different domain, and try to find the middle ground between the preferences of novices and expert users when summarising medical data while providing first aid. Finally, we want to compare the methodology presented here to a multi-objective optimisation approach (Fonseca and Flemming, 1993), where the preferences of each user group will be modelled as two different optimisation functions.

\section{Acknowledgements}

The research leading to this work has received funding from the EC's FP7 programme: (FP7/2011-14) under grant agreement no. 248765 (Help4Mood). 


\section{References}

Carole Ames. 1992. Classrooms: Goals, structures, and student motivation. Journal of Educational Psychology, 84(3):p261-71.

Scotty D. Craig, Arthur C. Graesser, Jeremiah Sullins, and Barry Gholson. 2004. Affect and learning: an exploratory look into the role of affect in learning with autotutor.

Carlos Fonseca and Peter Flemming. 1993. Genetic algorithms for multiobjective optimization: Formulation, discussion and generalization. In 5th International Conference on Genetic Algorithms.

Albert Gatt, Francois Portet, Ehud Reiter, James Hunter, Saad Mahamood, Wendy Moncur, and Somayajulu Sripada. 2009. From data to text in the neonatal intensive care unit: Using NLG technology for decision support and information management. AI Communications, 22: 153-186.

Dimitra Gkatzia, Helen Hastie, Srinivasan Janarthanam, and Oliver Lemon. 2013. Generating student feedback from time-series data using Reinforcement Learning. In 14th European Workshop in Natural Language Generation (ENLG).

Dimitra Gkatzia, Helen Hastie, and Oliver Lemon. 2014a. Comparing multi-label classification with reinforcement learning for summarisation of timeseries data. In 52nd Annual Meeting of the Association for Computational Linguistics (ACL).

Dimitra Gkatzia, Helen Hastie, and Oliver Lemon. 2014b. Finding Middle Ground? Multi-objective Natural Language Generation from Time-series data. In 14th Conference of the European Chapter of the Association for Computational Linguistics (EACL).

Jim Hunter, Yvonne Freer, Albert Gatt, Yaji Sripada, Cindy Sykes, and D Westwater. 2011. Bt-nurse: Computer generation of natural language shift summaries from complex heterogeneous medical data. American Medical Informatics Association, 18:621624.

Srinivasan Janarthanam and Oliver Lemon. 2010. Adaptive referring expression generation in spoken dialogue systems: Evaluation with real users. In 11th Annual Meeting of the Special Interest Group on Discourse and Dialogue (SIGDIAL).

Ian T. Jolliffe. 1982. A note on the use of principal components in regression. Journal of the Royal Statistical Society, Series C: 31(3):300-303.

Ian Jolliffe. 1986. Principal Component Analysis. Springer-Verlag.

Saad Mahamood and Ehud Reiter. 2011. Generating Affective Natural Language for Parents of Neonatal Infants. In 13th European Workshop in Natural Language Generation (ENLG).
Natalie K. Person, Roger J. Kreuz, Rolf A. Zwaan, and Arthur C. Graesser. 1995. Pragmatics and pedagogy: Conversational rules and politeness strategies may inhibit effective tutoring. Journal of Cognition and Instruction, 13(2):161-188.

Ehud Reiter and Robert Dale. 2000. Building natural language generation systems. Cambridge University Press.

Ehud Reiter, Roma Robertson, and Liesl Osman. 1999. Types of knowledge required to personalise smoking cessation letters. Artificial Intelligence in Medicine: Proceedings of the Joint European Conference on Artificial Intelligence in Medicine and Medical Decision Making.

Verena Rieser, Oliver Lemon, and Xingkun Liu. 2010. Optimising information presentation for spoken dialogue systems. In 48th Annual Meeting of the Association for Computational Linguistics (ACL).

Richard Sutton and Andrew Barto. 1990. Time derivative models of pavlovian reinforcement. Learning and Computational Neuroscience: Foundations of Adaptive Networks, pages 497-537.

Richart Sutton and Andrew Barto. 1998. Reinforcement learning. MIT Press.

Cynthia A. Thompson, Mehmet H. Goker, and Pat Langley. 2004. A personalised system for conversational recommendations. Journal of Artificial Intelligence Research, 21(1).

Marilyn Walker, Diane J Litman, Candace Kamm, and Alicia Abella. 1997. PARADISE: A framework for evaluating spoken dialogue agents. In 8 th conference on European chapter of the Association for Computational Linguistics (EACL).

Ingrid Zukerman and Diane Litman. 2001. Natural language processing and user modeling: Synergies and limitations. In User Modeling and UserAdapted Interaction, 11(1-2). 